\title{
Resuscitation of Asphyxic Newborn Infants with Room Air or $100 \%$ Oxygen
}

\author{
SIDDARTH RAMJI, SANJIV AHUJA, S. THIRUPURAM, TERJE ROOTWELT, GÖSTA ROOTH, AND \\ OLA DIDRIK SAUGSTAD \\ Department of Pediatrics, Maulana Azad Medical College, New Delhi-110002, India [S.R., S.A., S.T.] \\ Department of Pediatric Research. The National Hospital, Oslo, Norway [T.R., O.D.S.]; and Department of \\ Pediatrics, Uppsala University Hospital, Uppsala, Sweden [G.R.]
}

\begin{abstract}
To test the hypothesis that room air is superior to $100 \%$ oxygen when asphyxiated newborns are resuscitated, 84 neonates (birth weight $>999$ g) with heart rate $<80$ and/or apnea at birth were allocated to be resuscitated with either room air $(n=42)$ or $100 \%$ oxygen $(n$ $=42$ ). Serial, unblinded observations of heart rates at 1 , 3, 5, and $10 \mathrm{~min}$ and Apgar scores at $1 \mathrm{~min}$ revealed no significant differences between the two groups. At $5 \mathrm{~min}$, median (25th and 75th percentile) Apgar scores were higher in the room air than in the oxygen group $[8$ (7-9) versus $7(6-8), p=0.03$ ]. After the initial resuscitation, arterial partial pressure of oxygen, $\mathrm{pH}$, and base excess were comparable in the two groups. Assisted ventilation was necessary for $2.4(1.5-3.4) \mathrm{min}$ in the room air group and 3.0 (2.0-4.0) $\mathrm{min}$ in the oxygen group $(p=0.14)$. The median time to first breath was $1.5(1.0-2.0) \mathrm{min}$ in both the room air and oxygen groups $(p=0.59)$, and the time to first cry was $3.0(2.0-4.0) \mathrm{min}$ and $3.5(2.5-5.5) \mathrm{min}$ in the room air and oxygen groups, respectively $(p=0.19)$. Three neonates in the room air group and four in the oxygen group died in the neonatal period. At $28 \mathrm{~d}, 72$ of the 77 surviving neonates were available for follow-up (36 in each group), and none had any neurologic sequelae. This preliminary study did not provide conclusive evidence that room air is superior to $100 \%$ oxygen in the resuscitation of asphyxiated newborns, although it indicated that room air is as effective as $100 \%$ oxygen. Additional trials with increased numbers of patients are necessary before deciding whether room air or oxygen should be used in clinical practice. (Pediatr Res 34: 809-812, 1993)
\end{abstract}

\section{Abbreviations}

HIE, hypoxic-ischemic encephalopathy $\mathrm{PaO}_{2}$, arterial partial pressure of oxygen

$\mathrm{PaCO}_{2}$, arterial partial pressure of carbon dioxide

In the wake of the disasters with retinopathy of preterm infants in the early fifties, the oxygen concentrations in the incubators and the time supplementary oxygen was used were curtailed. Sjöstedt and Rooth (1) observed that preterm and term infants thrived well in incubators with only $15 \%$ oxygen. In recent years, it has become evident that oxygen radicals are produced in excess in the posthypoxic reoxygenation period (2-4) with the potential to increase tissue damage. Despite this, guidelines for the resuscitation of asphyxiated newborn infants advocate the use of

Received December 8, 1992; accepted July 20, 1993.

Correspondence: O. D. Saugstad, Department of Pediatric Research, The National Hospital, 0027 Oslo, Norway. supplementary oxygen, usually $100 \%$ oxygen $(5,6)$. To our knowledge, no clinical studies have tested this practice.

Saugstad has previously suggested that the use of $100 \% \mathrm{O}_{2}$ may be detrimental in the resuscitation of hypoxic newborn infants $(2,4,7,8)$. Recent experimental work from his group with newborn pigs suggests that room air is as effective as $100 \%$ oxygen (9-11). Given this background, we have felt it not only justified but also necessary to investigate whether resuscitation of newborn infants should be performed with room air instead of $100 \%$ oxygen. Furthermore, in many developing countries, supplementary oxygen is rarely available at all places and at all times, and it is important to find out whether resuscitation can be performed effectively even when oxygen is not at hand.

In the present preliminary study, we have investigated the short-term effects of resuscitating asphyxic newborn infants with either room air or $100 \%$ oxygen.

\section{MATERIALS AND METHODS}

The investigation was initiated and designed in Oslo and Uppsala and performed at the Department of Pediatrics, Maulana Azad Medical College, New Delhi, India. We tested the hypothesis that room air is superior to $100 \%$ oxygen when asphyxiated newborn infants are resuscitated. Primary outcome measures used for this preliminary study were 1) Apgar score and 2) heart rate, both at $5 \mathrm{~min}$. Secondary outcome variables were $\mathrm{PaO}_{2}, \mathrm{PaCO}_{2}$, and base excess at 10 and $30 \mathrm{~min}$ to assess posthypoxic respiratory and metabolic recovery. The study was also intended as a pilot experiment to calculate sample size requirements for later studies.

Sample size. The sample size was calculated on the basis of the first primary outcome measure. A trial of 72 babies (36 in each group) would have an $80 \%$ power to detect as statistically significant ( $p<0.05$, two-tailed test) a difference of 1 in mean Apgar score at $5 \mathrm{~min}$ if SD is 1.5 .

Enrollment criteria were 1 ) heart rate $<80 \mathrm{bpm}$ and/or apnea at birth justifying resuscitation, and 2) weight $>999 \mathrm{~g}$. Newborn babies with lethal anomalies, hydrops fetalis, or congenital cyanotic heart defects were excluded. Neonates born on even dates were allocated to the room air group, and those born on odd dates, to the oxygen group.

Protocol for ventilation. The neonates were bagged with an AMBU Infant Resuscitator (Ambu International Inc., Brøndby, Denmark) using a face mask and a ventilatory frequency of about $60 / \mathrm{min}$ for as long as it was needed. Neonates in the room air group who were cyanosed and/or bradycardic after $90 \mathrm{~s}$ were switched to $100 \%$ oxygen supplementation. One hundred percent oxygen was delivered by connecting a corrugated tube reservoir to the bag with an oxygen flow rate of at least $4 \mathrm{~L} / \mathrm{min}$.

Baseline parameters. Detailed baseline material, intrapartum, and neonatal variables were recorded in every newborn enrolled into the study. These included maternal age, parity, antenatal 
care received (regarded adequate if at least three hospital visits had been made in the last two trimesters), maternal diseases (hypertension, anemia, antepartum/accidental hemorrhage, or infection), presence of fetal distress (meconium stained liquor with or without abnormal fetal heart rate patterns $<100$ or $>180$ bpm), prolonged second-stage labor, mode of delivery, sedation, birth weight, and gestation.

Measurements. Heart rate (recorded by auscultation) at 0,1 , 3, 5, and $10 \mathrm{~min}$ and Apgar scores at 1 and $5 \mathrm{~min}$ were recorded. The times for the appearance of the first spontaneous breath and the first cry were noted, as well as the duration of assisted ventilation. There was no blinding of the observer recording these events.

Arterial blood samples were taken at 10 and $30 \mathrm{~min}$ from the right radial artery, and blood gases and acid base status were measured on an Eschweiler 2000 blood gas analyzer (Eschweiler, Kiel, Germany).

Short-term outcome. To evaluate short-term outcome, abnormal neurologic status at d $0-3,4-7$, and 28 , as well as neonatal deaths, were recorded. Disturbed neurologic status was regarded as present if the neonate was hypo- or hypertonic, had reflex responses (Moro, sucking, rooting) considered inappropriate for the neonate's gestation, or had convulsions. Clinical staging of HIE was by the method described by Sarnat and Sarnat (12). At 1 mo of age, head lifting in the supine position, smiling, and fixation, as well as the absence or presence of spasticity, were noted. The observer evaluating the neurologic status was blinded to the neonate's treatment allocation.

Ethical approval. Approval was obtained from the hospital's ethics committee.

Statistical analysis. Arterial blood gas and acid-base values were analyzed using $t$ test. Serial changes in heart rate were evaluated by analysis of variance for repeated measures. Duration of assisted ventilation, time to first breath and cry, and Apgar scores were analyzed by Mann-Whitney $U$ test. Discrete data were evaluated by $\chi^{2}$ or Fisher's exact test. A two-tailed $p<0.05$ was considered statistically significant (13).

\section{RESULTS}

Eighty-five neonates were initially enrolled into the study. One neonate in the room air group who was apneic with a heart rate of zero at birth could not be resuscitated and was declared a stillbirth. This baby was excluded from the study. Forty-two neonates were therefore available for analysis in each treatment group.

Baseline characteristics. Table 1 gives the important baseline maternal and intrapartum characteristics. The groups were comparable with respect to these variables.

Treatment protocol. Table 2 provides information on some relevant aspects of the treatment protocol. There were no statistically significant differences between the groups with regard to

Table 1. Baseline maternal and intrapartum characteristics

\begin{tabular}{lcc}
\hline & $\begin{array}{c}\text { Room air } \\
(n=42)\end{array}$ & $\begin{array}{c}\text { Oxygen } \\
(n=42)\end{array}$ \\
\hline Mean (SD) maternal age $(\mathrm{y})$ & $24.4(4.2)$ & $23.8(4.3)$ \\
Antenatal care received $[n(\%)]$ & $20(48)$ & $19(45)$ \\
Maternal disease [ $n(\%)]$ & $17(41)$ & $14(33)$ \\
Abnormal fetal heart rate & $11(26)$ & $18(43)$ \\
$\quad[n(\%)]^{*}$ & & \\
Meconium-stained liquor & $19(45)$ & $16(38)$ \\
$\quad[n(\%)]^{*}$ & & \\
Prolonged II stage [ $n(\%)]$ & $3(7)$ & $5(12)$ \\
Vaginal breech $[n(\%)]$ & $5(12)$ & $5(12)$ \\
Cesarean section $[n(\%)]$ & $10(24)$ & $9(21)$ \\
Mean (SD) birth weight $(\mathrm{g})$ & $2410(540)$ & $2410(660)$ \\
Mean (SD) gestation (wk) & $38.4(1.9)$ & $38.1(2.6)$ \\
\hline
\end{tabular}

* Twenty-seven neonates in each group had evidence of fetal distress.
Table 2. Details of treatment allocation and follow-up

\begin{tabular}{lcc}
\hline & $\begin{array}{c}\text { Room air } \\
(N)\end{array}$ & $\begin{array}{c}\text { Oxygen } \\
(N)\end{array}$ \\
\hline Infants enrolled & 42 & 42 \\
Ventilated by face mask only & 32 & 25 \\
Intubated at birth & 6 & 14 \\
Ventilated by face mask and & 4 & 3 \\
$\quad$ subsequently intubated & 6 & 0 \\
Crossed over to other group & 36 & 36 \\
Available for follow-up at 28 d & \\
\hline
\end{tabular}

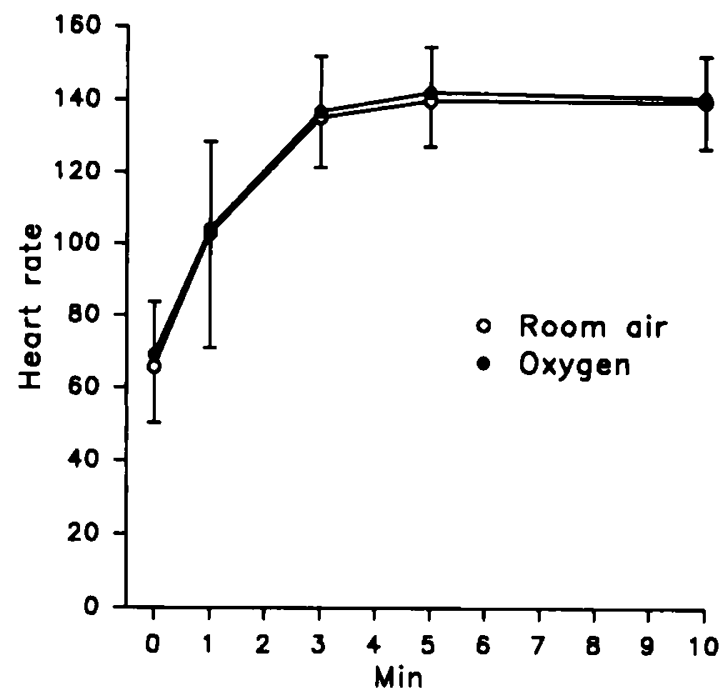

Fig. 1. Serial mean (SD) heart rate changes in room air $(n=42)$ and oxygen $(n=42)$ groups.

the number of neonates ventilated by mask, ventilated by endotracheal tube, or switched over from mask to subsequent endotracheal tube. At birth, intubation was carried out in six neonates in the room air group (all for meconium aspiration) and 14 in the oxygen group ( 12 for meconium aspiration and two for heart rates $<60 \mathrm{bpm}$ ). Among the rest who were initially ventilated with bag and mask, four in the room air group and three in the oxygen group were intubated between 1.5 and $3.0 \mathrm{~min}$ after birth for persistent bradycardia (heart rate $<100 \mathrm{bpm}$ ). Six neonates in the room air group failed to respond to resuscitation within $90 \mathrm{~s}$ and were switched over to $100 \%$ oxygen as per protocol. These six neonates were retained in the room air group for statistical analysis (intention to treat). Unfortunately, information on the number of neonates in the oxygen group who might have been cyanosed and bradycardic at $90 \mathrm{~s}$ is not available.

Heart rate and Apgar score. Heart rate (Fig. 1) was normalized as quickly in the room air as in the oxygen group (analysis of variance $p=0.86$ ). Apgar scores were similar in the room air and oxygen groups at $1 \mathrm{~min}$ [Median (25th and 75th percentile): $4(2-4)$ versus $4(3-4), p=0.97$ ], but at $5 \mathrm{~min}$, Apgar scores were higher in the room air group than in the oxygen group [8 (7-9) versus $7(6-8), p=0.03$ ]

Arterial blood gas and acid-base values. Blood gas values at 10 and $30 \mathrm{~min}$ after birth are presented in Table 3 . There were no differences in $\mathrm{PaO}_{2}, \mathrm{pH}$, or base excess. The room air group had a significantly lower mean (SD) $\mathrm{PaCO}_{2}$ at 30 min than the oxygen group [3.6 (1.1) versuis $4.3(1.5) \mathrm{kPa}, p<0.05$ ]. All neonates (except one in the room air group who was ventilated beyond 60 $\mathrm{min}$ ) were breathing spontaneously by $10 \mathrm{~min}$. Five neonates in the oxygen group at 10 and $30 \mathrm{~min}$ and two in the room air group at 30 min were receiving supplementary oxygen by hood for respiratory distress. At $10 \mathrm{~min}$, five neonates $(12 \%)$ in the room air group and eight (19\%) in the oxygen group had a $\mathrm{PaO}_{2}$ $>13.3 \mathrm{kPa}(100 \mathrm{~mm} \mathrm{Hg})$, whereas at $30 \mathrm{~min}$, seven $(17 \%)$ and $11(26 \%)$ neonates in the room air and oxygen groups, respec- 
Table 3. Arterial blood gas and acid-base status at 10 and 30 min after birth*

\begin{tabular}{lccc}
\hline & $\begin{array}{c}\text { Time } \\
(\mathrm{min})\end{array}$ & $\begin{array}{c}\text { Room air } \\
(n=42)\end{array}$ & $\begin{array}{c}\text { Oxygen } \\
(n=42)\end{array}$ \\
\hline $\mathrm{PaO}_{2}(\mathrm{kPa})$ & 10 & $10.1(3.7)$ & $10.1(3.3)$ \\
& 30 & $10.9(5.7)$ & $11.0(4.2)$ \\
$\mathrm{PaCO}_{2}(\mathrm{kPa})$ & 10 & $4.8(2.1)$ & $5.0(1.7)$ \\
$\mathrm{pH}$ & 30 & $3.6(1.1) \dagger$ & $4.3(1.5)$ \\
& 10 & $7.16(0.12)$ & $7.17(0.16)$ \\
Base excess (mmol/L) & 30 & $7.24(0.14)$ & $7.28(0.11)$ \\
& 10 & $-15.2(5.7)$ & $-13.6(6.6)$ \\
& 30 & $-13.0(6.1)$ & $-10.8(4.9)$ \\
\hline
\end{tabular}

* Values are given as mean (SD).

$\dagger p<0.05$ room air $v s$ oxygen group.

Table 4. Number of neonates with abnormal neurologic examination during the first week of life

\begin{tabular}{lccccc}
\hline & \multicolumn{3}{c}{ Room air } & & \multicolumn{2}{c}{ Oxygen } \\
\cline { 2 - 3 } \cline { 5 - 6 } & $\begin{array}{c}\text { Survivors } \\
(n=39)\end{array}$ & $\begin{array}{c}\text { Dead } \\
(n=3)\end{array}$ & $\begin{array}{c}\text { Survivors } \\
(n=38)\end{array}$ & $\begin{array}{c}\text { Dead } \\
(n=4)\end{array}$ \\
\hline Abnormal neurology & 4 & 3 & & 4 & 1 \\
HIE & 0 & 0 & & 1 & 0 \\
I & 1 & 0 & & 1 & 0 \\
II & 0 & 3 & & 0 & 1 \\
\hline
\end{tabular}

tively, had a $\mathrm{PaO}_{2}>13.3 \mathrm{kPa}$. These differences were not significant. Breathing room air, at $10 \mathrm{~min}$, four neonates in the

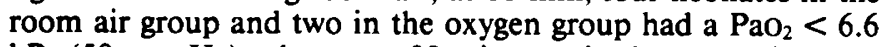
$\mathrm{kPa}(50 \mathrm{~mm} \mathrm{Hg})$, whereas at $30 \mathrm{~min}$, one in the room air group and five in the oxygen group had a $\mathrm{PaO}_{2}<6.6 \mathrm{kPa}$. These differences, too, were not significant.

Ventilatory efficacy. The median (25th and 75th percentile) duration of assisted ventilation was $2.4(1.5-3.4) \mathrm{min}$ in the room air group and $3.0(2.0-4.0) \mathrm{min}$ in the oxygen group ( $p=$ $0.14)$. The median time to first breath was $1.5(1-2)$ min and 1.5 $(1-2) \mathrm{min}$ in the room air and oxygen groups, respectively $(p=$ $0.59)$. The median time to first cry was $3.0(2.0-4.0) \mathrm{min}$ and $3.5(2.5-5.5) \mathrm{min}$ in the room air and oxygen groups, respectively $(p=0.19)$.

Neurologic abnormality and mortality (Table 4). Deaths occurred in three neonates in the room air group (all related to birth asphyxia) and four in the oxygen group (one related to asphyxia and three due to respiratory distress). The death occurred within the first $3 \mathrm{~d}$ of life (except one in the oxygen group who died at $6 \mathrm{~d}$ ). Seven neonates in the room air group and five in the oxygen group had an abnormal neurologic examination during the first week of life. Clinical evidence of HIE was observed in four neonates in the room air group and three in the oxygen group. All neonates who had HIE stage III died. At $28 \mathrm{~d}$, 36 infants in each group were available for follow-up, and all were neurologically normal.

\section{DISCUSSION}

The question of which oxygen concentration is necessary for successful resuscitation of asphyxic newborns has not been adequately studied, and to our knowledge no clinical trials have been reported. Clinical experience using room air and mouthto-mouth resuscitation have thus far been the only evidence to support the clinical efficacy of room air during resuscitation at birth.

In a study of newborn rabbits, Campbell et al. (14) observed that there were no significant differences in time to first gasp or survival whether room air or $100 \%$ oxygen was used. More recently, Rootwelt et al. (9) studied newborn piglets exposed to $8 \%$ oxygen until systolic blood pressure had fallen to $20 \mathrm{~mm} \mathrm{Hg}$ (with a base excess of about $-28 \mathrm{mmol} / \mathrm{L}$ ). It was observed that resuscitation with room air normalized blood pressure, heart rate, metabolic acidosis, and plasma hypoxanthine concentration as quickly as when $100 \%$ oxygen was used. Neuropathologic examinations after $4 \mathrm{~d}$ did not reveal any significant differences between the groups. Similar results were obtained by the same group in slightly older piglets (10).

In the present study, the clinical recovery as judged by duration of assisted ventilation, heart rates, Apgar scores, and time to onset of spontaneous respiratory effort were comparable between the two groups, as were the changes in the blood gas and acidbase parameters. The lower Apgar score in the $100 \%$ oxygen group after $5 \mathrm{~min}$ should be regarded with some caution because the observers were not blinded to the treatment allocation. The low mean base excess values in both the groups suggest a moderate to severe character of the asphyxic insult. The $\mathrm{pH}$ and base excess values were not normalized after $30 \mathrm{~min}$. These results are consistent with the studies of Oliver et al. (15) in human neonates and Rootwelt et al. (9) in piglets, which indicate that it may take 30-180 min to normalize base excess after asphyxia. The significantly lower $\mathrm{PaCO}_{2}$ in the room air group at $30 \mathrm{~min}$ may be a compensation by the neonate for the slightly larger base deficit in this group, inasmuch as by this time all neonates, except one, were breathing spontaneously. The neonatal mortality and neurologic abnormalities were comparable in the two groups. However, because of the small numbers in the present study, $95 \%$ confidence intervals are very wide. These results must therefore be validated by studies with larger sample sizes. To have an $80 \%$ chance to detect a $50 \%$ reduction in mortality (from 10 to $5 \%$ ), a total sample size of 870 babies is necessary.

Six neonates did not respond to room air within $90 \mathrm{~s}$ according to the criteria set in the protocol. The response to resuscitation after $90 \mathrm{~s}$ was not recorded systematically in the oxygen group. However, mean heart rate normalized at least as quickly during the first $60 \mathrm{~s}$ (before any infants were switched to $100 \%$ oxygen) in the room air as in the oxygen group, indicating that these "room air failures" do not represent a true difference between the treatments.

This study suggests that if the ventilation is sufficient, room air adequately oxygenates most neonates with birth weights $>$ $999 \mathrm{~g}$ with moderate to severe perinatal asphyxia, as observed by the low proportion of neonates who had $\mathrm{PaO}_{2}<6.7 \mathrm{kPa}$ on room air after 10 and $30 \mathrm{~min}$. Thus, adequate ventilation is probably more important than oxygen supplementation during resuscitation. However, some newborns with severely impaired lung function may not be adequately oxygenated with room air. An alternative approach could therefore be to use an oxygen blender to deliver oxygen concentrations according to clinical response and/or pulse oximetry. However, routines for resuscitations should be as simple as possible, and pulse oximeters are not always reliable (16).

An important question is whether posthypoxic oxygen supplementation has a significant effect on oxygen radical production. Both superoxide production by xanthine oxidase (17) and brain hydrogen peroxide production (18) increase with increasing arterial oxygen tensions, but in vivo animal studies after brain ischemia have provided conflicting results $(19,20)$. It is possible that sudden normoxic reoxygenation results in near maximal oxygen radical production and that to reduce this a more gradual reintroduction of oxygen may be necessary $(21-23)$. The present study did not address this issue, and because blood gases were not analyzed during resuscitation, the risks of initial hyperoxia or hypoxia cannot be evaluated.

The results of this preliminary study did not provide evidence that room air is superior to $100 \%$ oxygen in the resuscitation of asphyxiated newborns, but it indicates that room air may be as effective as $100 \%$ oxygen. Because of the limited number of patients enrolled in the present study, the results must be validated in larger multicenter trials before deciding whether or not 
room air should be recommended routinely for neonatal resuscitation.

\section{REFERENCES}

1. Sjöstedt S, Rooth G 1957 Low oxygen tension in the management of newborn infants. Arch Dis Child 32:397-400

2. Saugstad OD, Aasen AO 1980 Plasma hypoxanthine concentration in pigs. A prognostic aid in hypoxia. Eur Surg Res 12:123-129

3. McCord JM 1985 Oxygen-derived free radicals in postischemic tissue injury. N Engl J Med 312:159-163

4. Saugstad OD 1990 Oxygen toxicity in the neonatal period. Acta Paediatr Scand 79:881-892

5. Milner AD 1991 Resuscitation of the newborn. Arch Dis Child 66:66-69

6. American Heart Association 1992 Guidelines for cardiopulmonary resuscitation and emergency cardiac care. JAMA 268:2276-228

7. Saugstad OD 1985 Oxygen radicals and pulmonary damage. Pediatr Pulmonol 1:167-175

8. Saugstad OD 1989 The oxygen radical disease in neonatology. Indian J Pediatr 56:585-593

9. Rootwelt T, Löberg EM, Moen A, Oyasaeter S, Saugstad OD 1992 Hypoxemia and reoxygenation with $21 \%$ or $100 \%$ oxygen in newborn pigs: changes in blood pressure, base deficit and hypoxanthine and brain morphology. Pediatr Res 32:107-113

10. Poulsen JP, Oyasaeter S, Saugstad OD 1993 Hypoxanthine, xanthine and uric acid in newborn pigs during hypoxemia followed by resuscitation with room air or 100\% oxygen. Crit Care Med 21:1058-1065

11. Rootwelt T, Odden J-P, Hall C, Ganes T, Saugstad OD 1993 Cerebral blood flow and evoked potentials during reoxygenation with $21 \%$ or $100 \% \mathrm{O}_{2}$ in newborn pigs. J Appl Physiol 75 (in press)
12. Sarnat HB, Sarnat MS 1976 Neonatal encephalopathy following fetal distress. A clinical and electroencephalographic study. Arch Neurol 33:696-705

13. Altman DG 1990 Practical Statistics for Medical Research. Chapman and Hall, London

14. Campbell AGM, Cross KW, Dawes GS, Hyman AI 1966 A comparison of air and oxygen in a hyperbaric chamber or by positive pressure ventilation in the resuscitation of newborn rabbits. J Pediatr 68:153-163

15. Oliver TK, Demis JA, Bates GD 1961 Serial blood gas tensions and acid base balance during the first hour of life in human infants. Acta Paediatr Scand $50: 346-360$

16. Baeckert P, Bucher HU, Fallenstein F, Fanconi S, Huch R, Duc G 1987 Is pulse oximetry reliable in detecting hyperoxemia in the neonate? Adv Exp Med Biol 220:165-169

17. Fridovich I 1970 Quantitative aspects of the production of superoxide anion radical by milk xanthine oxidase. J Biol Chem 245:4053-4057

18. Yusa T, Beckman JS, Crapo JD, Freeman BA 1987 Hyperoxia increases $\mathrm{H}_{2} \mathrm{O}_{2}$ production by brain in vivo. J Appl Physiol 63:353-358

19. Mickel HS, Vaishnav YN, Kempski O, von Lubitz D, Weiss JF, Feuerstein G 1987 Breathing $100 \%$ oxygen after global brain ischaemia in Mongolian gerbils results in increased lipid peroxidation and increased mortality. Stroke $18: 426-430$

20. Agardh CD, Zhang H, Smith ML, Siesjö BK 1991 Free radical production and ischemic brain damage: influence of postischemic oxygen tension. Int J Devl Neurosci 9:127-128

21. Perry MA, Wadhwa SS 1988 Gradual reintroduction of oxygen reduces reperfusion injury in cat stomach. Am J Physiol 254:G366-G372

22. Korthuis RJ, Smith JK, Carden DL 1989 Hypoxic reperfusion attenuates postischemic microvascular injury. Am J Physiol 256:H315-H319

23. Orendácová J, Marsala M, Marsala J 1991 The blood-brain barrier permeability in graded postischaemic spinal cord reoxygenation injury in rabbits. Neurosci Lett 128:143-146 